Health \& Medicine | Nandu Thalange

\section{Flexibility is key in treating children with type 1 diabetes}

People with type 1 diabetes
cannot produce their own
insulin, leading to uncontrolled
blood sugar levels. To survive,
they must receive insulin
treatment. A recently
developed, long-lasting insulin
called degludec offers a unique
level of flexibilitity. This means
that it is particularly suitable
for treating children and young
people with type 1 diabetes. Dr
Nandu Thalange, an expert in
childhood diabetes at Al Jalila
Children's Speciality Hospital,
Al Jaddaf, Dubai, UAE, has
created several "fictionalised"
case studies drawn from real
life to help other doctors to use
degludec effectively.

nsulin is a vital hormone. When you eat or drink, the process of digestion converts carbohydrates into simple
sugars - mainly glucose. Glucose passes into the blood, where it is transported around the body. The pancreas contains beta cells, which release insulin according to the amount of glucose in the blood If glucose is low, no insulin is made, but if you have guzzled a litre of lemonade, your beta cells go into overdrive and produce enormous amounts of insulin. Normally, this metabolic dance happens completely unconsciously.

In diabetes, this system breaks down. The commonest form of diabetes is type 2 , which accounts for about $90 \%$ of all those affected. Only around $8-10 \%$ of people with diabetes have the

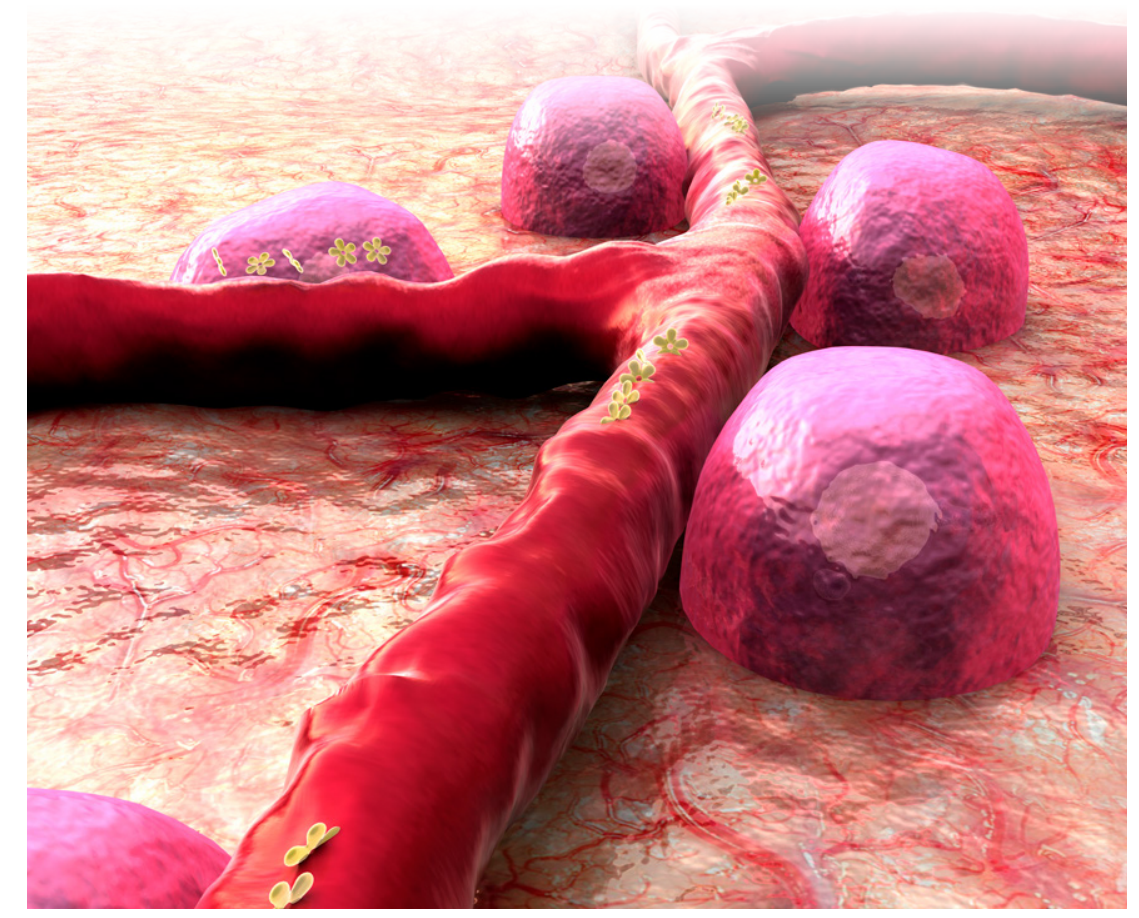

In type 1 diabetes, the beta cells are atlacked by the immune system. On acrical number of beta cells have succumber little warning diabetes evolve In the absence of insulin, the body misreads the situation: it interprets the absence of insulin as a sign of dangerously low glucose levels. The liver starts to manufacture glucose As glucose levels rise, the kidneys can no longer conserve glucose and it spills into the urine, acting like a diuretic. Excess urine production quickly leads dehydration, thirst and rapid weight loss.

Type 1 diabetes can occur at any age, but most commonly affects children and adolescents. The younger you For the more aggressive the disease. For childran, type 1 drabetes must be The number of new cases of type 1 diabetes is increasing in young people, and children are getting it at a younger age. One study suggests that the rate of new cases of diabetes in children will triple between 2010 and 2050

\section{THE BLOOD SUGAR} BALANCING ACT

People with type 1 diabetes cannot produce their own insulin. To survive they must receive insulin treatment.

Insulin treatment is a complicated balancing act between diet, exercise an insulin. An imbalance may lead to high will eventually hyperglycaemia - which will eventually damage the vital organs, nerves and blood vessels, leading to
serious long-term complications. Too further increasing the blood sugar level.

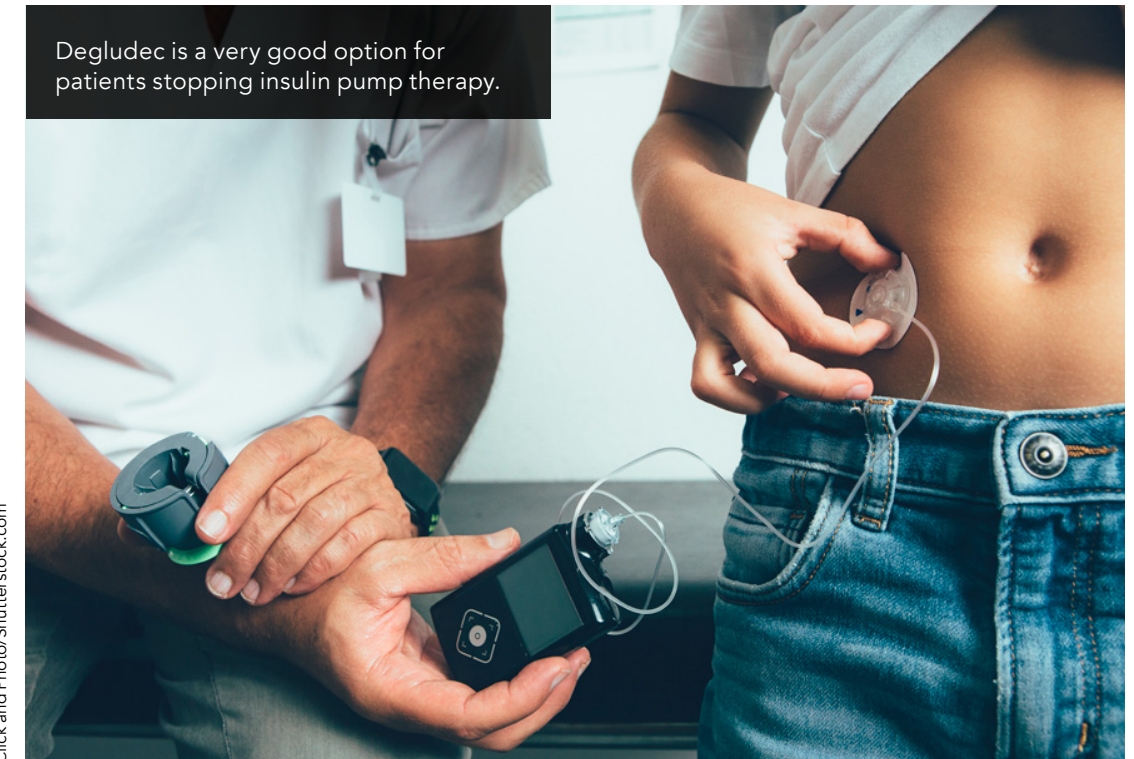

Physically and psychologically, children may respond differently to treatments when compared to adults.

much insulin leads to low blood sugar, or hypoglycaemia. Every person with type 1 diabetes is trained to recognise and treat hypoglycaemia, or "hypos". to identify - especially at night - and may have serious consequences such as a coma or seizures.

Unfortunately, intensive treatmen is associated with more frequent hypoglycaenia. The arrival of the first genetically altered insulins - or insul frequent hypoglycaemia.

THE CHALLENGES OF TREATING DIABETES IN CHILDREN Without insulin treatment, type 1 there available. Most people with type 1 diabetes take insulin via injection. The first part of this treatment is a basal insulin. a "background insulin designed to keep blood sugar levels stable between meals. Additional "bolus" insulin injections are required for meals and snacks.

Physically and psychologically, children respond differently to treatment when

vary more in their day-to-day lifestyle in diet and physical activity, for example. They are also more susceptible to ilness. These factors introduce a level of unpredictability that can make treating same time every day, for example.

Dr Nandu Thalange of Al Jalila Children's Speciality Hospital, Dubai, is a paediatric in childhood diabetes Nandu believes that the tretment of Nandu believes chat the treatment of type 1 diabetes in

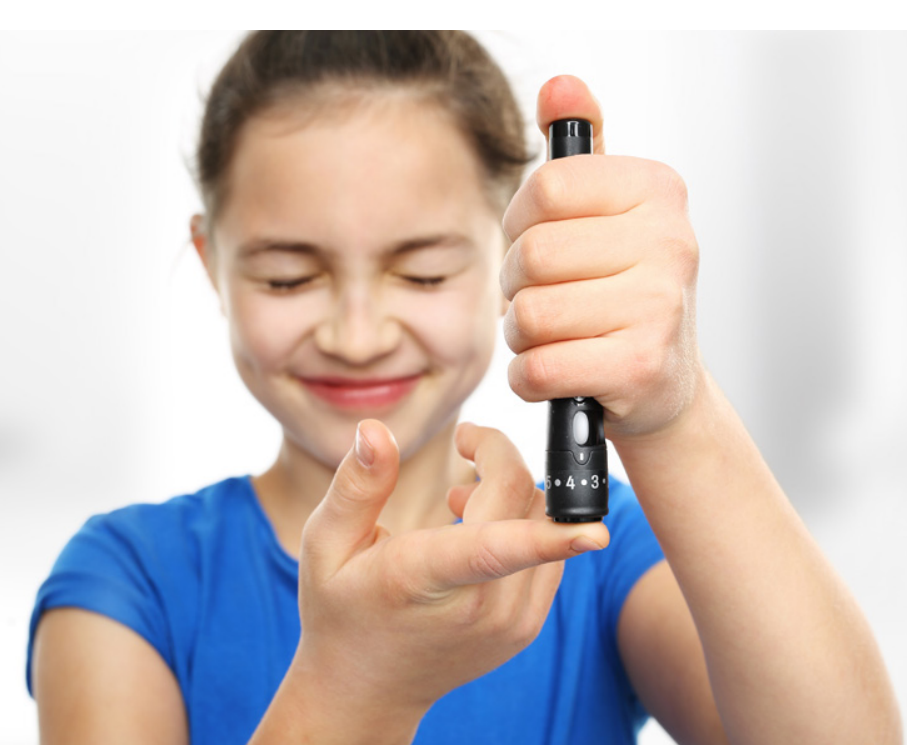
type 1 diabetes in children tricky; many many existing treatments do not offer. degludec was launched. This new type of hasal insulin offers exactly the type of flexibility and control that is needed by young people with diabetes.

DEGLUDEC: A NEW TYPE OF LONG-LASTING INSULIN

Degludec is currently the only longlasting insulin approved for treating diabetes in very young children. Degludec has a half-life (the time it takes for the concentration of the maintening absorbed by the body, - a condition called "steady state". This means that clad steady stat. This or even duplicated doses - are much less of a proble than with other thpes of basal insulin. Another advantage of achieving steady state means that there is always some insulin onboard diabetic ketoacidosis.

Nandu played a leading role in the BEGIN Young 1 trial that led to degludec being approved for the treatment of diabetes in childhood. After recognising n unmet need for guidance on safe and effective use of degludec, Nandu hopes on how to make best use of its "steady state biochemistry, particularly when (reating children. To this " end, Nandu " case gludec effectively. drug in the body to reduce by $50 \%$ ) of core than 24 hours, and is slowly and preventing a serious condition called to assist other physicians by advising 
LONG-LASTING INSULIN

\section{OFFERS FLEXIBILITY}

One key case study describes how to

and adolescents can vary greatly in the

dose of insulin needed for effective blood

sugar control. factors such as age, BMI

and puberty should all be considered. For

daily dose. This can then be gradually

increased until blood glucose stabilises.

Once degludec has reached steady state

the dose can be adjusted.

Doctors might choose to switch patients to degludec to take advantage of the needs to be taken once daily, which process distressing Dege injection process distesting Degludec is a good parent has difficulty keeping to a rigid teenager who forgets his or her degludec dose at bedtime can take it in the up, with no harm done.

One particular case study neatly demonstrates just how useful the be. Nandu explains:

Daisy was diagnosed with diabetes on given an insulf called glarghe, but she night and after exercise.

Daisy's parents found the strain of diabetes unbearable. A continuous glucose monitor reduced the stress of frequent glucose checks overnight, or also suggested an insulin pump to prevent low blood sugar, but Daisy was a very boisterous, active girl, and her parents felt it would be too much for her.

Daisy's dad travelled often for work Once, after coming home late, mum forgot the glargine and didn't realise this reason, it is important to begin with

a fraction - say $40 \%$ - of the expected in the blood stream, in around three days, dings unique properties. Degludec only dosing schedule, as the dose timing can be varied widely from day to day The morning (or afternoon!) when they wake unique properties of degludec can her 4th birthday, while seriously ill with diabetic ketoacidosis. At frst, Daisy was and her mum worked shifts. This made giving the glargine on time very difficult. until Daisy started vomiting the next

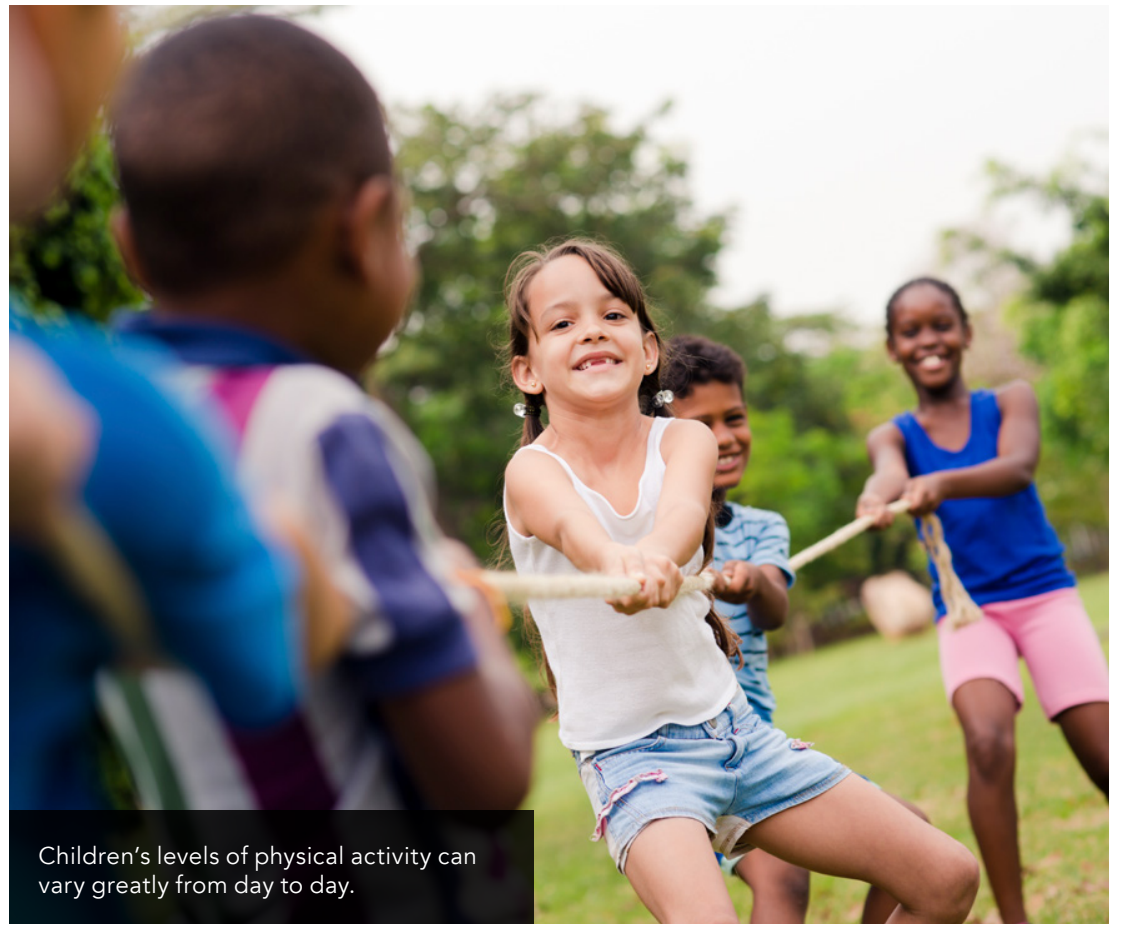

Degludec offers exactly the type of flexibility that is needed in treating young people.

gave Daisy the glargine and then dad,

$\begin{array}{ll}\text { gave Daisy the glargine and then dad, } & \text { therapy. In an example given by Nandu, } \\ \text { arriving home late, gave another dose } & \text { a teenager did not notice that his pump }\end{array}$ a teenager did not notice that his pump her glucose drinks.

Their doctor suggested changing to degludec. Unlike other insulins, it can not cause a major prouble dose' does not cause a major problem - just skip degludec and had fewer hypos. The diabetes was still tough to live with but the flexibility and peace of mind the degludec gave was a great relief.

The other main system for managing diabetes is an insulin pump. These are pocket-sized devices that deliver insulin continuously throughout the day and night and work well for many people with type 1 diabetes. These devices are usually combined with a continuous blood glucose monitor. Some will even adjust the insulin supply accordingly. Pump users tend to need less insulin an levels. However, pumps are not suitab fril, necestient and can sometimes had detached from his body during and hey had to stay up all night feeding be given up to 16 hours early or late. have better-controlled blood glucose weep, which led to a hospital admission with diabetic ketoacidosis. Some teenagers, with very active lifestyles, are "sible" treatment

THE FUTURE OF

\section{TETES TREATMENT?}

Despite the slightly higher cost of

egludec compared with other basal

cullins, is lower dosing means ir is

Its greater stability gives it a shelf-life of

56 days, whereas most insulins have to

be discarded after 30 days in use. If one factors in reduced ketosis - the precursor to ketoacidosis - the cost savings are

dramatic.

Nandu and his colleagues believe, based on their broad experience, that degludec is a highly effective treatment for young people with type 1 diabetes. The unique flexibility of the drug means that it has the potential to improve the health, prognosis, and quality of life for many with this serious, life-long condition.

\section{3a) Behind the Research} Dr Nandu Thalange

E: nandu.thalange@ajch.ae T: +971 042811000

\section{Research Objectives}

Pr Nandu Thalange has created a narrative review of the clinical use of degludec in children with Type 1 diabetes.

\section{Detail}

Bio

Nandu Thalange graduated from King's College docrnology. After completing his training, he took up Norfolk and Norwich University Hospital in East Anglia until he left in 2017 to move to Dubai, to take up a post at Al Jalila Children's Hospita.

Nandu has been an investigator in a number of investigator for the international multi-centre trials that led to the approval of insulin degludec in children. He is an honorary Senior Lecturer at the University of East Anglia, UK and adjunct Professor of Pediatrics at ohammed Bin Rashid University, Dubai.

Funding

For developed insulin degludec. The BEGIN program regulatory authorities around the world. Novo Nordisk supported the development of the paper on which this article is based.

Collaborators

My fellow authors, Thomas Danne and Torben Biester, and the many children who taught us.

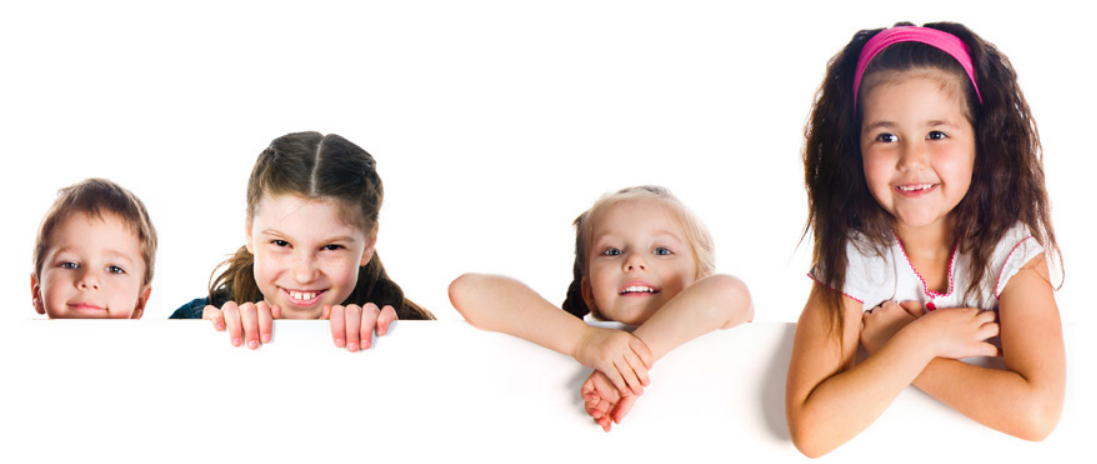
research studies in diabetes, including being chief

\section{References}

Thalange, N., Biester, T., Danne, T., 2019. Clinical use of degludec in children and adolescents with T1D: a narrative review with fictionalized case reports. Diabetes

\section{Personal Response}

In your experience, how has degludec improved the quality of life of children and young people with type 1 diabetes?

II Insulin degludec offers real benefits for patients f amilies - better, more stable background insulin with freedom to dose when convenient ( \pm 16 hou
with lower rates of low blood sugar and diabetic ketoacidosis, in a single daily iniection. In my opinion is the best basal insulin there is, and has really tangible benefits for patients.

Recently, I presented a small pilot study of degludec ("honey most patients achieve a sustained remission biochemistry of degludec is reducing the deme the damaged beta cells of the pancreas, by controlling glucose toxicity. In practical terms, this means really good glucose control, which is sustained for a longer period. Landmark studies have shown that good benefits, ey 30 ylabetes has decisive and enduring complications and deaths. from diagnosis and our preliminary results appear 